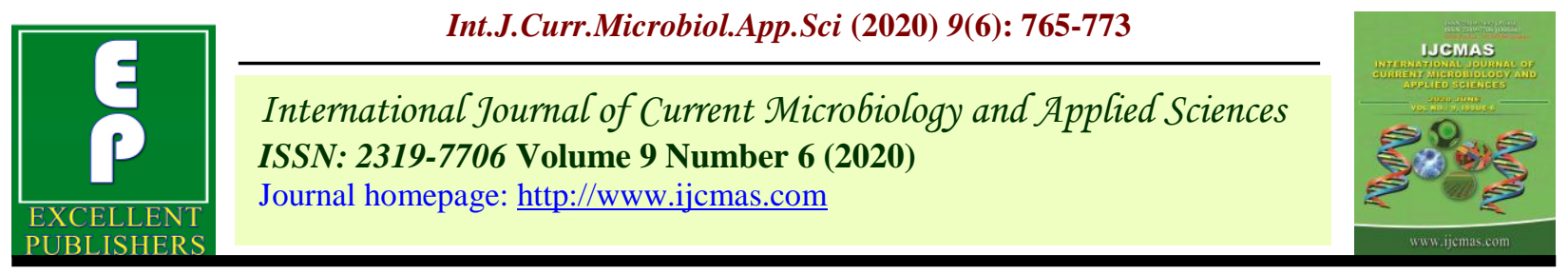

Original Research Article

https://doi.org/10.20546/ijcmas.2020.906.098

\title{
Scheduling of Irrigation in Cauliflower (Brassica oleracea var. botrytis L.) under Mid Hill Conditions of Himachal Pradesh
}

\author{
Manju Kumari* and Meera Devi \\ Department of Agriculture, Maharishi Markandeshwar University, Sadopur Ambala, India \\ *Corresponding author
}

\begin{abstract}
A B S T R A C T
\section{Keywords}

Yield, Root mass density and root volume density, Uptake, WUE, CU

Article Info

Accepted:

15 May 2020

Available Online:

10 June 2020

The present investigation entitled Scheduling of irrigation in cauliflower (Brassica oleracea var. botrytis L.) under mid hill conditions of Himachal Pradesh was conducted during 2011-12 and 2012-13 at the experimental farm of Department of Soil Science and Water Management, Dr. Y.S. Parmar University of Horticulture and Forestry, Nauni- Solan, H.P. The experiment was laid out in Randomized Block Design with eight treatments and three replications i.e. $3 \mathrm{~cm}$ irrigation at IW/CPE ratio i.e. $1.0\left(\mathrm{~T}_{1}\right), 0.8\left(\mathrm{~T}_{2}\right)$ and $0.6\left(\mathrm{~T}_{3}\right)$; at $0.4\left(\mathrm{~T}_{4}\right), 0.5\left(\mathrm{~T}_{5}\right)$ bar tensiometric suction; at $25\left(\mathrm{~T}_{6}\right)$ and $50\left(\mathrm{~T}_{7}\right)$ per cent depletion of available water in $0-30 \mathrm{~cm}$ depth; farmer's practice $\left(\mathrm{T}_{8}\right)$ i.e. $5 \mathrm{~cm}$ irrigation at 12-15 days interval depending upon the rainfall). The irrigation schedules had significant effect on crop growth and productivity of cauliflower under $T_{1}, T_{4}$ and $T_{6}$ irrigation treatments. The crop yield increased by, 29.5, 30.1 and 32.2 per cent over $\mathrm{T}_{3}$ and 25.5 and 26.0 and 28.1 per cent over $\mathrm{T}_{8}$ irrigation schedule. Schedule $\mathrm{T}_{1}$ required less quantity of irrigation water $(18.9 \mathrm{~cm}$ and $21.9 \mathrm{~cm})$ compared to $\mathrm{T}_{6}(30.9 \mathrm{~cm}$ and $36.9 \mathrm{~cm})$ and $\mathrm{T}_{4}(27.9 \mathrm{~cm}$ and $33.9 \mathrm{~cm})$ without any significant reduction in yield and gave higher WUE and B:C ratio. Thus, all these three schedules were equally good. Keeping in view the productivity, WUE and $\mathrm{B}: \mathrm{C}$ ratio, $\mathrm{T}_{1}$ irrigation schedule was found efficient and economical.
\end{abstract}

\section{Introduction}

Cauliflower (Brassica oleracea var. botrytis L.) is an important winter vegetable crops of mid hill region of Himachal Pradesh. Its commercial cultivation as an off season vegetable crop for remunerative returns, more demand in the markets of neighbouring plains and improved nutritional awareness of people have attracted the farmers to bring large area under its cultivation. However, the productivity of crop is low and variable owing to erratic distribution of rain fall, poor irrigation facilities and unscientific use of scarce irrigation water. The farmers are largely dependent upon rains and are unable to achieve higher yield of better quality under rainfed conditions for sustaining the crop 
productivity. This situation is further deteriorated by erratic distribution and meager winter rains in the state which creates a condition of water scarcity during the active growth period of vegetable crops. This crop is sensitive to moisture stress at different growth stages and stress at any critical stage reduces its quality and productivity. Irrigation scheduling is one of the most important tools for overcoming water stress and developing best water management strategies for water scarce and irrigated areas. It is essential for the judicious use of water and maximizing crop yields. Irrigation scheduling is the use of water management strategies to prevent over application of water while minimizing the yield loss due to water shortages or drought stress.

Characteristics of the crop are taken into account, for achieving higher and stable yields of irrigated crop (Kanton et al., 2003; Pejic et al., 2008). The proper irrigation schedule consists of applying irrigation to the crop at the "right time" and in "right amount". On the other hand improper timing and insufficient water application can result in moisture stress, reduced nutrient uptake and lower water use efficiency (Olczyk et al., 2000).

\section{Materials and Methods}

Field study was conducted at the experimental farm of Department of Soil Science and Water Management, Dr. Y.S. Parmar University of Horticulture and Forestry, Nauni- Solan, H .P during 2011-12 and 201213. Farm yard manures and recommended doses of fertilizers in the form of $\mathrm{N}, \mathrm{P}_{2} \mathrm{O}_{5}$ and $\mathrm{K}_{2} \mathrm{O}$ nutrients were added as per the schedule of the experiment in the form of calcium ammonium nitrate (CAN), single superphosphate (SSP) and muriate of potash (MOP).the experiment consisted of eight treatments replicated three times in a randomaized block design viz., $\mathrm{T}_{1}: 3 \mathrm{~cm}$ irrigation at IW/CPE $=1.0, \mathrm{~T}_{2:} 3 \mathrm{~cm}$ irrigation at $\mathrm{IW} / \mathrm{CPE}=0.8, \mathrm{~T}_{3}: 3 \mathrm{~cm}$ irrigation at $\mathrm{IW} / \mathrm{CPE}=0.6, \mathrm{~T}_{4}: 3 \mathrm{~cm}$ irrigation at $0.4 \mathrm{bar}$ tensiometric suction, $\mathrm{T}_{5}: 3 \mathrm{~cm}$ irrigation at 0.5 bar tensiometric suction, $\mathrm{T}_{6}: 3 \mathrm{~cm}$ irrigation at 25 percent depletion of available water, $\mathrm{T}_{7}$ : $3 \mathrm{~cm}$ irrigation at 50 percent depletion of available water, $\mathrm{T}_{8}$ : Farmer's practice (heavy irrigation i.e. $5 \mathrm{~cm}$ at $12-15$ days interval depending upon the rainfall). The whole dose of FYM, $\mathrm{P}$ and $\mathrm{K}$ fertilizers were applied at the time of field preparation. The nitrogen fertilizers was applied in three split doses, first dose at the time of transplanting and second dose one month after transplanting and third dose at the time of flowering. Yield was recorded after harvest of cauliflower. RV and RMD were calculated using the formula:

Root volume density $=$

Volume of roots by displacement method

\section{Volume of core}

After completing above, the roots were then transferred to a filter paper and pressed gently in its folds to remove imbibed water. The roots were then dried in oven at 60 $\pm 5^{\circ} \mathrm{C}$ till a constant weight and finally, the dried weight was taken and RMD was calculated as follows:

Root mass density $=\frac{\text { Dry weight of roots }}{\text { Volume of core }}$

\section{Consumptive use of water}

Consumptive use of water was computed as per the method given by Dastane (1972):

Consumptive use $(\mathrm{cm})=$ Profile water use $(\mathrm{cm})+$ effective rainfall $(\mathrm{cm})+$ ground water 
contribution $(\mathrm{cm})$. The depth of water table was more than $25 \mathrm{~m}$ below the surface soil throughout the period of experimentation. Hence, the ground water contribution was considered as nil.

\section{Profile water use}

The profile water use was worked out by the following formula:

$$
\mathrm{d}=\sum_{\mathrm{i}=0} \frac{\mathrm{M}_{1}{ }^{\mathrm{i}}-\mathrm{M}_{2}{ }^{\mathrm{i}}}{100} \times \mathrm{ASi} \text { x Di }+ \text { ER }
$$

Where,

$\mathrm{d}=$ Moisture deficit in the root zone

$\sum_{i=0}^{n}=$ Summation of ' $n$ ' number of layers

$\mathrm{M}_{1}{ }^{\mathrm{i}}=$ Soil moisture in $\mathrm{i}^{\text {th }}$ layer of profile 24 hours after irrigation

$\mathrm{M}_{2}{ }^{\mathrm{i}}=$ Soil moisture in $\mathrm{i}^{\text {th }}$ layer of profile on the day just before the next irrigation

$\mathrm{ASi}=$ bulk density of $\mathrm{i}^{\text {th }}$ layer $\left(\mathrm{g} \mathrm{cm}^{-3}\right)$

Di $=$ Depth of $i^{\text {th }}$ layer $(\mathrm{cm})$ and

$\mathrm{ER} \quad=$ Effective rainfall $(\mathrm{cm})$

\section{Water requirement}

Total water requirement was computed as :

Water requirement $=$ Sum total of irrigation water applied + Effective rainfall

\section{Water use efficiency (WUE)}

The WUE was calculated for each treatment with the help of following formula:

$$
\begin{aligned}
& \text { WU E }\left(\mathrm{kg} \mathrm{ha}^{-1} \mathrm{~mm}^{-1}\right)= \\
& \text { Yield (kg/ha) }
\end{aligned}
$$

Irrigation water applied (mm)

\section{Effective rainfall}

Effective rainfall was calculated by balance sheet method as suggested by Gupta et al., (1972)

\section{Results and Discussion}

The two years Pooled result of the growth and quality parameters of cauliflower at harvest stage are given in Table.1. The results revealed that the irrigation scheduling was formed to affect significantly all the plant parameters of cauliflower. Among irrigation schedules $\mathrm{T}_{6}$ recorded higher value of curd equatorial diameter $(15.0 \mathrm{~cm})$, curd polar diameter $(9.3 \mathrm{~cm})$, stalk length $(5.1 \mathrm{~cm})$ and vitamin $\mathrm{C}\left(52.2 \mathrm{mg} 100 \mathrm{~g}^{-1}\right)$ over $\mathrm{T}_{3}$, but was found to be at par with $\mathrm{T}_{4}$ and $\mathrm{T}_{6}$ irrigation schedule. It can be attributed to the better ferforfiratce ${ }^{o n f}$ various growth parameters due to optimum soil moisture and nutrient availability which ensured balanced water and nutrient supply throughout the crop season. Similar findings on onion crop have been reported by Qadir et al., (2005) and Bagali et al., (2012). Among these schedules stalk length increases due to due to frequent irrigations and quantum of water applied under $\mathrm{T}_{6}$ (eight to ten irrigations and 30.9 to $36.9 \mathrm{~cm}$ quantity of water applied) during the crop season. Vitamin $\mathrm{C}$ could be attributed to optimum moisture and nutrient availability creating favorable soil environment Shivakumar et al., (2011). Irrigation schedules had non-significant effect on Curd shape index.

\section{Growth parameters}

The result pertaining to curd wt and curd yield are given in Table 2. The results revealed that the effect of irrigation schedules was significant. Significantly higher curd weight was recorded in $\mathrm{T}_{6}\left(918.2 \mathrm{~g} \mathrm{plant}^{-1}\right)$ when $3 \mathrm{~cm}$ irrigation was applied at 25 per 
cent depletion of available water over in $T_{3}$ (694.2 $\mathrm{g} \mathrm{plant}^{-1}$ ) irrigation schedule. The irrigation schedules had significant impact both on curd weight and curd yield. Curd yield significantly influenced by irrigation schedules. The treatments $\mathrm{T}_{6}(264.4 \mathrm{q} / \mathrm{ha}), \mathrm{T}_{4}$ (259.0q/ha) and $T_{1}(260.2 \mathrm{q} / \mathrm{ha})$ which were at par with each other registered significantly higher curd yield over $\mathrm{T}_{3}$ (199.9 q/ha) and $\mathrm{T}_{8}$ (206.3 q/ha) irrigation schedules. This may be due to the optimum soil moisture content and nutrient availabilities and better root growth. The favorable effects of irrigation in increasing the yield of crops have also been reported by Kadam et al., (2006) and Imtiyaz et al., (2009).

The highest root mass density $\left(1.26 \mathrm{gm}^{-3} \times 10^{-3}\right)$ was observed in $\mathrm{T}_{6}$ irrigation schedule which was at par with $\mathrm{T}_{4}\left(1.04 \mathrm{gm}^{-3} \times 10^{-3}\right)$ irrigation schedules over $\mathrm{T}_{3}\left(0.63 \mathrm{gm}^{-3} \times 10^{-3}\right)$. It might be due to optimum soil moisture contents in rhizosphere which were highest and near FC
(23.05 \& 21.85) during the crop growth season encouraging better proliferation and elongation of roots. Several workers have documented the favorable effects of irrigation schedules on root growth especially in vegetable crops. The frequent irrigations to okra, wheat and Lolium perenne (L.) crops at (25 per cent depletion of available water) produced comparatively higher root growth due to optimum moisture availability (Mandal et al., 2003). Irrigation schedules had a significant effect on RVD in cauliflower. The schedule $\mathrm{T}_{6} \mathrm{RVD}$ of $\left(5.18 \mathrm{~m}^{3} \mathrm{~m}^{-3} \times 10^{-3}\right)$ which gave significantly higher RVD over $T_{3}$ $\left(2.81 \mathrm{~m}^{3} \mathrm{~m}^{-3} \times 10^{-3}\right)$ and $\mathrm{T}_{8}\left(4.29 \mathrm{~m}^{3} \mathrm{~m}^{-3} \times 10^{-3}\right)$ irrigation schedules. Higher RVD in $T_{6}$ irrigation schedule could be attributed to the better moisture conditions per cent in the root zone and increased the nutrient availability . These results are also in consonance with those Webber et al., (2006) and Zoolech et al., (2011).

Table.1 Effect of irrigation schedule on Curd equatorial diameter, curd polar diameter, curd shape index, stalk length and vitamin $\mathrm{C}$

\begin{tabular}{|c|c|c|c|c|l|}
\hline Treatments & $\begin{array}{c}\text { Curd } \\
\text { equatorial } \\
\text { diameter }(\mathbf{c m})\end{array}$ & $\begin{array}{c}\text { Curd polar } \\
\text { diameter } \\
(\mathbf{c m})\end{array}$ & $\begin{array}{c}\text { Curd shape } \\
\text { index }\end{array}$ & $\begin{array}{l}\text { Stalk } \\
\text { length } \\
(\mathbf{c m})\end{array}$ & $\begin{array}{l}\text { Vitamin-C } \\
\left(\mathbf{m g} \mathbf{1 0 0 g}^{-\mathbf{1}}\right)\end{array}$ \\
\hline $\mathbf{T}_{\mathbf{1}}$ & Pooled & Pooled & Pooled & Pooled & Pooled \\
\hline $\mathbf{T}_{\mathbf{2}}$ & 14.5 & 9.0 & 0.5 & 5.0 & 52.1 \\
\hline $\mathbf{T}_{\mathbf{3}}$ & 12.8 & 8.3 & 0.5 & 4.7 & 46.0 \\
\hline $\mathbf{T}_{\mathbf{4}}$ & 12.4 & 7.6 & 0.6 & 4.3 & 45.9 \\
\hline $\mathbf{T}_{\mathbf{5}}$ & 14.6 & 9.2 & 0.5 & 4.9 & 52.0 \\
\hline $\mathbf{T}_{\mathbf{6}}$ & 13.3 & 7.9 & 0.6 & 4.5 & 47.6 \\
\hline $\mathbf{T}_{\mathbf{7}}$ & 15.0 & 9.3 & 0.5 & 5.1 & 52.2 \\
\hline $\mathbf{T}_{\mathbf{8}}$ & 13.7 & 8.5 & 0.6 & 4.6 & 50.4 \\
\hline $\mathbf{C D}_{(\mathbf{0 . 0 5}}$ & 13.6 & 8.2 & 0.6 & 4.4 & 50.9 \\
\hline
\end{tabular}


Table.2 Effect of irrigation schedules on curd weight, curd yield root mass density and root volume density of cauliflower

\begin{tabular}{|c|c|c|c|c|}
\hline Treatments & $\begin{array}{c}\text { Curd } \\
\text { weight (g } \\
\left.\text { plant }^{-1}\right)\end{array}$ & $\begin{array}{c}\text { Curd yield } \\
\text { (q/ha) }\end{array}$ & $\begin{array}{c}\text { Root Mass } \\
\text { Density } \\
\left(\mathrm{g} \mathrm{m}^{-3} \times 10^{-3}\right)\end{array}$ & $\begin{array}{c}\text { Root volume } \\
\text { Density } \\
\left(\mathbf{m}^{3} \mathrm{~m}^{-3} \times 10^{-3}\right)\end{array}$ \\
\hline $\mathbf{T}_{1}$ & 903.5 & 260.2 & 0.76 & 3.41 \\
\hline $\mathbf{T}_{2}$ & 816.5 & 235.1 & 0.74 & 3.32 \\
\hline $\mathbf{T}_{3}$ & 694.2 & 199.9 & 0.63 & 2.81 \\
\hline $\mathbf{T}_{4}$ & 899.5 & 259.0 & 1.09 & 4.89 \\
\hline $\mathbf{T}_{5}$ & 776.7 & 223.6 & 0.94 & 4.20 \\
\hline $\mathbf{T}_{6}$ & 918.2 & 264.4 & 1.26 & 5.18 \\
\hline $\mathbf{T}_{7}$ & 885.0 & 246.2 & 1.04 & 4.35 \\
\hline $\mathbf{T}_{8}$ & 716.5 & 206.3 & 0.96 & 4.29 \\
\hline $\mathrm{CD}_{(0.05)}$ & 13.71 & 9.31 & 0.13 & 0.61 \\
\hline
\end{tabular}

\begin{tabular}{|c|c|c|c|c|}
\hline Treatments & $\begin{array}{l}\text { Curd weight } \\
\left.\text { (g plant }^{-1}\right)\end{array}$ & $\begin{array}{c}\text { Curd yield } \\
\text { (q/ha) }\end{array}$ & $\begin{array}{c}\text { Root Mass } \\
\text { Density } \\
\left(\mathrm{g} \mathrm{m}^{-3} \times \mathbf{1 0}^{-3}\right)\end{array}$ & $\begin{array}{c}\text { Root volume } \\
\text { Density }\left(\mathrm{m}^{3} \mathrm{~m}^{-3} \mathbf{x} 10\right. \\
\left.{ }^{\prime}\right)\end{array}$ \\
\hline $\mathbf{T}_{1}$ & 903.5 & 260.2 & 0.76 & 3.41 \\
\hline $\mathbf{T}_{2}$ & 816.5 & 235.1 & 0.74 & 3.32 \\
\hline $\mathbf{T}_{3}$ & 694.2 & 199.9 & 0.63 & 2.81 \\
\hline $\mathbf{T}_{4}$ & 899.5 & 259.0 & 1.09 & 4.89 \\
\hline $\mathbf{T}_{5}$ & 776.7 & 223.6 & 0.94 & 4.20 \\
\hline $\mathbf{T}_{6}$ & 918.2 & 264.4 & 1.26 & 5.18 \\
\hline $\mathbf{T}_{7}$ & 885.0 & 246.2 & 1.04 & 4.35 \\
\hline $\mathbf{T}_{8}$ & 716.5 & 206.3 & 0.96 & 4.29 \\
\hline $\mathrm{CD}_{(0.05)}$ & 13.71 & 9.31 & 0.13 & 0.61 \\
\hline
\end{tabular}

Table.3 Effect of irrigation schedule on nutrient uptake in cauliflower

\begin{tabular}{|l|c|c|c|c|c|c|}
\hline \multirow{2}{*}{ Treatments } & \multicolumn{3}{|c|}{ Pooled (Uptake) } & \multicolumn{3}{c|}{ Pooled (Uptake) } \\
\cline { 2 - 7 } & $\mathbf{N}$ & $\mathbf{P}$ & $\mathbf{K}$ & $\mathbf{C a}$ & $\mathbf{M g}$ & $\mathbf{S}$ \\
\hline $\mathbf{T}_{\mathbf{1}}$ & 47.7 & 6.8 & 44.6 & 21.7 & 9.3 & 5.5 \\
\hline $\mathbf{T}_{\mathbf{2}}$ & 42.3 & 8.4 & 39.9 & 21.6 & 8.5 & 5.9 \\
\hline $\mathbf{T}_{\mathbf{3}}$ & 37.9 & 6.6 & 35.6 & 19.6 & 6.4 & 4.8 \\
\hline $\mathbf{T}_{\mathbf{4}}$ & 46.8 & 10.0 & 42.7 & 26.0 & 8.4 & 7.1 \\
\hline $\mathbf{T}_{\mathbf{5}}$ & 42.5 & 8.4 & 39.7 & 22.5 & 7.2 & 4.8 \\
\hline $\mathbf{T}_{\mathbf{6}}$ & 49.3 & 9.6 & 45.4 & 26.6 & 10.1 & 5.4 \\
\hline $\mathbf{T}_{\mathbf{7}}$ & 47.1 & 9.5 & 44.3 & 24.1 & 9.3 & 5.9 \\
\hline $\mathbf{T}_{\mathbf{8}}$ & 35.0 & 5.5 & 34.4 & 17.2 & 6.9 & 4.2 \\
\hline $\mathbf{C D}_{(\mathbf{0 . 0 5}}$ & $\mathbf{1 . 8 5}$ & $\mathbf{2 . 6 7}$ & $\mathbf{4 . 8 3}$ & $\mathbf{3 . 6 2}$ & $\mathbf{1 . 7 7}$ & $\mathbf{2 . 0 5}$ \\
\hline
\end{tabular}


Table.4 Water use components of cauliflower as influenced by irrigation schedules

\begin{tabular}{|c|c|c|c|c|c|c|c|c|c|c|}
\hline \multirow[t]{2}{*}{$\begin{array}{l}\text { Treatmen } \\
\text { ts }\end{array}$} & \multicolumn{2}{|c|}{$\begin{array}{l}\text { Irrigation water } \\
\text { applied (cm) }\end{array}$} & \multicolumn{2}{|c|}{$\begin{array}{c}\text { Effective } \\
\text { rainfall }(\mathbf{c m})\end{array}$} & \multicolumn{2}{|c|}{$\begin{array}{c}\text { Total water } \\
\text { requirement } \\
(\text { IWA+E.R) }(\mathrm{cm})\end{array}$} & \multicolumn{2}{|c|}{$\begin{array}{c}\text { Consumptive use } \\
\text { (cm) }\end{array}$} & \multicolumn{2}{|c|}{$\begin{array}{l}\text { Water use efficiency } \\
\quad\left(\mathrm{kg} \mathrm{ha}^{-1} \mathrm{~mm}^{-1}\right)\end{array}$} \\
\hline & 2011-12 & $\begin{array}{c}2012- \\
13\end{array}$ & $\begin{array}{c}2011- \\
12\end{array}$ & $\begin{array}{c}2012- \\
13\end{array}$ & 2011-12 & $\begin{array}{c}\text { 2012- } \\
13\end{array}$ & $\begin{array}{c}\text { 2011- } \\
12\end{array}$ & 2012-13 & 2011-12 & 2012-13 \\
\hline $\mathbf{T}_{1}$ & $\begin{array}{l}* 18.9 \\
(4)\end{array}$ & $\begin{array}{c}21.9 \\
(5)\end{array}$ & 4.7 & 5.0 & $\begin{array}{c}23.6 \\
* *(-31.5)\end{array}$ & $\begin{array}{c}26.9 \\
(-7.8)\end{array}$ & 15.3 & 17.7 & $\begin{array}{c}110.6 \\
* * *(11.0)\end{array}$ & $\begin{array}{l}96.3 \\
(9.6)\end{array}$ \\
\hline $\mathbf{T} 2$ & $\begin{array}{c}15.9 \\
(3)\end{array}$ & $\begin{array}{c}18.9 \\
(4)\end{array}$ & 7.2 & 7.4 & $\begin{array}{c}23.1 \\
(-33.0)\end{array}$ & $\begin{array}{c}26.3 \\
(-9.9)\end{array}$ & 14.7 & 18.7 & $\begin{array}{l}103.4 \\
(10.2)\end{array}$ & $\begin{array}{l}87.9 \\
(8.7)\end{array}$ \\
\hline $\mathbf{T}_{3}$ & $\begin{array}{c}12.9 \\
(2)\end{array}$ & $\begin{array}{c}15.9 \\
(3)\end{array}$ & 8.4 & 10.6 & $\begin{array}{c}21.3 \\
(-38.2)\end{array}$ & $\begin{array}{c}26.5 \\
(-9.2)\end{array}$ & 14.8 & 20.0 & $\begin{array}{l}95.1 \\
(9.5)\end{array}$ & $\begin{array}{l}74.4 \\
(7.4)\end{array}$ \\
\hline $\mathbf{T 4}$ & $\begin{array}{c}27.9 \\
(7)\end{array}$ & $\begin{array}{l}33.9 \\
(9)\end{array}$ & 3.5 & 3.2 & $\begin{array}{c}31.4 \\
(-8.9)\end{array}$ & $\begin{array}{c}37.1 \\
(27.0)\end{array}$ & 16.0 & 21.2 & $\begin{array}{l}82.2 \\
(8.1)\end{array}$ & $\begin{array}{l}74.0 \\
(7.3)\end{array}$ \\
\hline T5 & $\begin{array}{c}24.9 \\
(6)\end{array}$ & $\begin{array}{c}27.9 \\
(7)\end{array}$ & 3.8 & 4.4 & $\begin{array}{c}28.7 \\
(-16.8)\end{array}$ & $\begin{array}{c}32.3 \\
(10.6)\end{array}$ & 16.6 & 17.7 & $\begin{array}{l}80.1 \\
(8.0)\end{array}$ & $\begin{array}{l}67.0 \\
(6.7)\end{array}$ \\
\hline$T_{6}$ & $\begin{array}{c}30.9 \\
(8)\end{array}$ & $\begin{array}{l}36.9 \\
(10)\end{array}$ & 3.1 & 4.6 & $\begin{array}{l}34.0 \\
(-1.4)\end{array}$ & $\begin{array}{c}41.5 \\
(42.1)\end{array}$ & 17.8 & 27.3 & $\begin{array}{l}77.9 \\
(7.7)\end{array}$ & $\begin{array}{l}63.5 \\
(6.3)\end{array}$ \\
\hline $\mathbf{T}_{7}$ & $\begin{array}{c}24.9 \\
(6)\end{array}$ & $\begin{array}{c}30.9 \\
(8)\end{array}$ & 6.5 & 4.2 & $\begin{array}{c}31.4 \\
(-8.9)\end{array}$ & $\begin{array}{c}35.1 \\
(20.2)\end{array}$ & 16.9 & 19.2 & $\begin{array}{l}79.8 \\
(7.9)\end{array}$ & $\begin{array}{l}68.9 \\
(6.8)\end{array}$ \\
\hline $\mathbf{T}_{8}$ & $\begin{array}{c}29.9 \\
(5)\end{array}$ & $\begin{array}{c}24.9 \\
(4)\end{array}$ & 4.6 & 4.3 & 34.5 & 29.2 & 15.8 & 14.7 & $\begin{array}{l}60.8 \\
(6.0)\end{array}$ & $\begin{array}{l}69.4 \\
(6.9)\end{array}$ \\
\hline
\end{tabular}

Figures in parenthesis are the number of irrigation applied *

Figures in parenthese are the (\%) water saving **

Figures in parenthese are the water use efficiency in $\left(\mathrm{kg} \mathrm{m}^{-3}\right) * * *$

Table.5 Cost economics of different irrigation schedules in cauliflower

\begin{tabular}{|c|c|c|c|c|}
\hline Treatments & $\begin{array}{c}\text { Total cost of } \\
\text { cultivation } \\
\text { (Rs/ha) }\end{array}$ & $\begin{array}{c}\text { Gross Income } \\
\text { (Rs/ha) }\end{array}$ & $\begin{array}{c}\text { Net Returns } \\
\text { (Rs/ha) }\end{array}$ & $\begin{array}{c}\text { B:C } \\
\text { Ratio }\end{array}$ \\
\hline $\mathbf{T}_{\mathbf{1}}$ & $58,638.5$ & 260200 & 201561.5 & $3.43: 1$ \\
\hline $\mathbf{T}_{\mathbf{2}}$ & $58,338.5$ & 235100 & 176761.5 & $3.02: 1$ \\
\hline $\mathbf{T}_{\mathbf{3}}$ & $58,038.5$ & 199900 & 141861.5 & $2.44: 1$ \\
\hline $\mathbf{T}_{\mathbf{4}}$ & $60,138.5$ & 259000 & 198861.5 & $3.30: 1$ \\
\hline $\mathbf{T}_{\mathbf{5}}$ & $58,938.5$ & 223600 & 164661.5 & $2.79: 1$ \\
\hline $\mathbf{T}_{\mathbf{6}}$ & $60,438.5$ & 264400 & 203961.5 & $3.37: 1$ \\
\hline $\mathbf{T}_{\mathbf{7}}$ & $59,538.5$ & 246200 & 186661.5 & $3.13: 1$ \\
\hline $\mathbf{T}_{\mathbf{8}}$ & $59,238.5$ & 206300 & 147061.5 & $2.48: 1$ \\
\hline
\end{tabular}




\section{Nutrient uptake}

The $\mathrm{T}_{6}$ irrigation schedule showed significantly higher uptake of $\mathrm{N}\left(43.3 \mathrm{~kg} \mathrm{ha}^{-1}\right)$ over $\mathrm{T}_{8}\left(35.0 \mathrm{~kg} \mathrm{ha}^{-1}\right)$ schedule and $\mathrm{T}_{4}$ showed higher uptake of $\mathrm{P}\left(10.0 \mathrm{~kg} \mathrm{ha}^{-1}\right)$ over $\mathrm{T}_{8}(5.5$ $\mathrm{kg} \mathrm{ha}^{-1}$ ) treatment. The $\mathrm{T}_{6}$ irrigation schedule showed higher uptake of $\mathrm{K}\left(45.4 \mathrm{~kg} \mathrm{ha}^{-1}\right), \mathrm{Ca}$ $\left(26.6 \mathrm{~kg} \mathrm{ha}^{-1}\right)$ and $\mathrm{Mg}\left(10.1 \mathrm{~kg} \mathrm{ha}^{-1}\right)$ over $\mathrm{T}_{8}$ $33.6 \mathrm{~kg} \mathrm{ha}^{-1}, 16.8 \mathrm{~kg} \mathrm{ha}^{-1}$ and $6.9 \mathrm{~kg} \mathrm{ha}^{-1}$ schedule and $\mathrm{S}$ uptake was highest under $\mathrm{T}_{4}$ $\left(7.1 \mathrm{~kg} \mathrm{ha}^{-1}\right)$ over $\mathrm{T}_{8}\left(4.2 \mathrm{~kg} \mathrm{ha}^{-1}\right)$ schedule. The higher uptake of nutrients recorded under $\mathrm{T}_{6}$ and $\mathrm{T}_{4}$ irrigation schedules might be due to optimum moisture conditions resulting in better growth of roots (Table 3) and there by nutrient uptake similar findings on maize, bean and sunflower have been reported by Murthy and Reddy, 2013.

\section{Irrigation water applied and effective rainfall}

The data pertaining to water applied and effective rainfall as influenced by different irrigation schedules are given in table 4 . During both years highest quantity of irrigation water was applied in $\mathrm{T}_{6}$ irrigation schedule and minimum recorded under $\mathrm{T}_{3}$ irrigation schedule. Whereas data on effective rain fall (ER) calculated during both the seasons under different irrigation schedules have been presented in Table 4. During first year of study, ER ranged from $3.1-8.4 \mathrm{~cm}$ under different irrigation schedules. Whereas highest $(8.4 \mathrm{~cm})$ ER was recorded under $\mathrm{T}_{3}$ irrigation schedule in which only two irrigations were applied during the period of crop and lowest $(3.1 \mathrm{~cm})$ ER under $\mathrm{T}_{6}$ irrigation schedule in which eight irrigations were applied. Similarly during second year of study highest $(10.6 \mathrm{~cm})$ ER was recorded under $\mathrm{T}_{3}$ irrigation schedule in which 3 irrigations were applied during the crop growth period and lowest $(3.2 \mathrm{~cm})$ ER was recorded under $\mathrm{T}_{4}$ irrigation schedule in which nine irrigations were applied.

\section{Water requirement, consumptive use and water use efficiency}

On an average basis among, $\mathrm{T}_{1}, \mathrm{~T}_{4}$ and $\mathrm{T}_{6}, \mathrm{~T}_{6}$ irrigation schedule recorded highest water requirement during both the years followed by $\mathrm{T}_{4}$ and $\mathrm{T}_{1}$ schedule but $\mathrm{T}_{1}$ irrigation schedule saved maximum amount of water i.e (20.7\%) followed by $\mathrm{T}_{4}(7.5 \%)$ and $\mathrm{T}_{6}(18.5$ $\%)$ irrigation schedule. Irrigation schedule $\mathrm{T}_{1}$ found to be superior over other schedule. The value of consumptive use of water were 17.8, 27.3 and 16.6,17.3 in T6 and T7 treatment, respectively. This would imply that the $\mathrm{CU}$ of water increase with the increase in level of irrigation as expected. This may also be due to the fact that surface layer of the soil remained wet for the longer duration under higher frequency of irrigation thus creating conditions for evaporation of water and absorption at higher rate as compared to the lower frequency of irrigation (Gulati et al., 2001; Raskar and Bhoi, 2003). The mean WUE recorded under T1 treatment (110.0, $96.3 \mathrm{~kg} \mathrm{ha}^{-1} \mathrm{~mm}^{-1}$ ) during both the years as it decreased with increasing level of irrigation. This may be due to greater loss of water through evapo-transpiration in frequently irrigated crop and without much reduction in yield with fairly lower water use (Sunder Singh 2001; Mahdi et al., 2003; Shiva Kumar et al., 2011;)

\section{Benefit-cost ratio}

Worked out for different irrigation schedules has been presented in table 5. A perusal of data reveals that maximum gross income was recorded in $\mathrm{T}_{6}$ (Rs 264400) irrigation schedule followed by $\mathrm{T}_{1}$ (Rs 260200), $\mathrm{T}_{4}$ (Rs 259000) and minimum (Rs 199900) under $T_{3}$ irrigation schedule. Similarly, net returns was maximum (Rs 203961.5) under $\mathrm{T}_{6}$ followed by $\mathrm{T}_{1}$ (Rs 201561.5), $\mathrm{T}_{4}$ (Rs 198861.5) and minimum (Rs 141861.5) under $\mathrm{T}_{3}$ irrigation 
schedule. The highest $\mathrm{B}: \mathrm{C}$ ratio $(3.43: 1)$ was worked out in treatment $\mathrm{T}_{1}$ which was rated as the most profitable and cost effective schedule followed by $\mathrm{T}_{6}(3.37: 1)$ which produced maximum yield. Hence, these three irrigation schedules were equally effective and anyone of these irrigation schedules can be used for maximizing the cauliflower yield.

\section{References}

Bagali A N, Patil H B, Guled M B and Patil R V. 2012. Effect of scheduling of drip irrigation on growth yield and water use efficiency of onion (Allium cepa L.). Karnataka Journal of Agricultural Sciences. 25(1): 116-119

Dastane N G. 1972. A practical manual for water use research. Pune, India: Navbharat Publication Mandir.

Gulati J M L, Lenka D and Paul J C. 2001. Moisture extraction pattern phasic water use and phasic growth in ground nut (Arachis hypogaea) under varying moisture regimes and ground water table condition. Indian Journal of Agronomy. 46(2): 287-291.

Gupta S K, Tejwani K S and Rambabu. 1972. Effective rainfall of Dehradun under irrigated condition. Symp. on soil and water management, ICAR held at Hissar, March 11-13. 1969. Pp 62-70

Imtiyaz M, Rongmei A and Singh V.2009. Proceedings of the $10^{\text {th }}$ International Agricultural Engineering Conference, Bangkok, Thailand, 7-10 December. Role of agricultural engineering in advent of changing global landscape.

Jackson M L. 1973. Soil Chemical Analysis. Printice Hall, India Pvt. Ltd., New Delhi

Kadam U S, Gorantiwar S D, Kadam S A, Gurav G B and Patil H M. 2006. Effects of different moisture regimes on yield potential of onion (Allium cepa L.) under microsprinkler irrigation system. Journal of Maharashtra Agriculture
University. 31(3): 342-345.

Kanton R A, Abbey L and Gbene R H. 2003. Irrigation schedule affects (Allium cepa L) growth, development and yield. Journal of Vegetable Production. 9(1): 3-11.

Mahdi M, Kaisi A and Yin X.2003. Effect of nitrogen rate, irrigation rate and plant population on corn yield and water use efficiency. Agronomy Journal. 95:14751482.

Mandal K G, hati K M, Mishra A K, Ghosh P K and Bandyopadhyay KK.2003. Root density and water use efficiency of wheat as affected by irrigation and nutrient management. Journal of Agriculture Physics. 3 (1\&2): 49-55.

Olczyk T, Regalado R, Li Y C and Jordan R. 2000. Usefulness of tensiometers for scheduling irrigation for tomatoes grown on rocky calcareous soils in Southern Florida. Proceedings of the Florida State Horticulture Society. 113: 239-242.

Pejic B, Gvozdanovic Varga J, Vasic M, Maksimovic L and Milic S. 2008. Yield and evapotranspiration of onion depending on different pre-irrigation soil moisture. (In Serbian) A Periodical of Science Research Field and Vegetable Crops. 44: 195-202.

Piper C S. 1966. Soil Chemical Analysis. Asia Publishing House, Bombay, 408 p.

Quadir M, Boulton A, Ekman J, Hickey M and Hoogers R. 2005. Influence of drip irrigation on onion yield and quality. IREC Farmers Newsletter, No. 170, 29 31 Australia

Raskar B S and Bhoi P G. 2003. Response of summer groundnut (Arachis hypogea L.) to irrigation regimes and mulching. Indian Journal of Agronomy. 48(3): 523-527.

Shivakumar H K, Ramachandrappa B K, Nanjappa H V and Mudalagiriyappa. 2011. Effects of phenophase based 
irrigation schedules on growth, yield and quality of baby corn (Zea mays L.). Agricultural Sciences. 2\&3: 267-272.

Sundarsingh S D.2001. Effect of irrigation regimes and nitrogen levels on growth yield and quality of baby corn. The Madras Agricultural Journal. 88:367370.

Webber H A, Madramootoo CA, Bourgault m, Horst M G, Stulina G and Smith D L. 2006. Water use efficiency of common bean and green gram grown using alternate furrow and deficit irrigation. Agricultural Water Management. 86 (3): 259-268.

Zoolech H H, Jahansooz M R, Yunusa I, Hosseini S B M, M R Chaichi and Jafari A A. 2011. Effect of alternate irrigation on root divided Foxtail Millet (Setaria italica). Australian Journal of Crop Science. 5(2): 205-213.

\section{How to cite this article:}

Manju Kumari and Meera Devi. 2020. Scheduling of Irrigation in Cauliflower (Brassica oleracea var. botrytis L.) under Mid Hill Conditions of Himachal Pradesh. Int.J.Curr.Microbiol.App.Sci. 9(06): 765-773. doi: https://doi.org/10.20546/ijcmas.2020.906.098 